\title{
Radio Emission from Supernova Remnants
}

\author{
By RICHARD G. STROM
}

Netherlands Foundation for Research in Astronomy, Radiosterrenwacht, Dwingeloo, The Netherlands

Most of the supernova remnants known in the Galaxy have only been detected at radio frequencies. The reason for this is absorption in the Galactic plane at both optical and X-ray wavelengths. All available evidence suggests that the shock fronts which accompany supernova remnants accelerate enough cosmic rays to $\mathrm{GeV}$ energies to produce readily detectable radio emission. This is fortunate, for it enables us to study remnants throughout the Galactic disk, although existing catalogues may be anywhere from 50 to $90 \%$ incomplete. Cosmic rays and the magnetic fields in which they gyrate are the essential ingredients for producing the synchrotron radiation which is observed at radio frequencies. Various methods for estimating magnetic field strengths can be applied to a small number of remnants, and produce values not far from those based upon equipartition between the energy contents of particles and fields. From this, the particle energy content is derived for a number of objects.

\section{Introduction}

If we could view the heavens with radio eyes, then the majestic sweep of the Galactic disk would dominate the large scale structure we see. This radio version of the Milky Way has greater symmetry about a more dominant Sagittarius than its optical counterpart, as can be seen in the fine $408 \mathrm{MHz}$ map of Haslam et al (1982). The main reason for this difference is simply dust, for although both stars and the cosmic rays responsible for decimeter radio emission are similarly distributed in the Galaxy, great clouds of the stuff obscure our optical vision, especially toward the Galactic center.

Returning to the radio view of things, if we sharpen our image of the Galactic plane to bring out more structural detail, improving the $1^{\circ}$ resolution of the $408 \mathrm{MHz}$ map by an order of magnitude (linearly), then some of the nonthermal radio emission becomes resolved into extended, by and large shell-like sources (e.g. Reich et al, 1990): supernova remnants (SNRs). Like the diffuse emission which dominates low frequency maps, the known SNRs in the Galaxy (e.g. Green, Appendix to these proceedings) lie near the equator, concentrated toward its central region (Fig. 1).

The shock waves produced by a supernova explosion heat the ambient gas to X-ray temperatures and simultaneously accelerate cosmic rays to $\mathrm{GeV}$ energies. This last fact is a fortunate turn of fate, for the X-ray emission, like the light from stars (or SNRs for that matter), is heavily absorbed by the interstellar medium. The energetically far less significant cosmic rays, corkscrewing their way through weak ambient magnetic fields, emit synchrotron radiation at radio frequencies which can traverse the entire Galactic disk essentially unattenuated. The existence of the majority of Galactic SNRs is known only from their radio emission.

In the remainder of this brief review, my goal will be to arrive at estimates for the cosmic ray and magnetic contents of typical SNRs, for they constitute the essential building blocks from a radio point of view. Along the way, I will touch upon SNR phenomenology and the morphological diversity to be found. Distances and their determination are also essential to deriving the physical parameters required, and finally methods for estimating magnetic field strengths round off the story. 


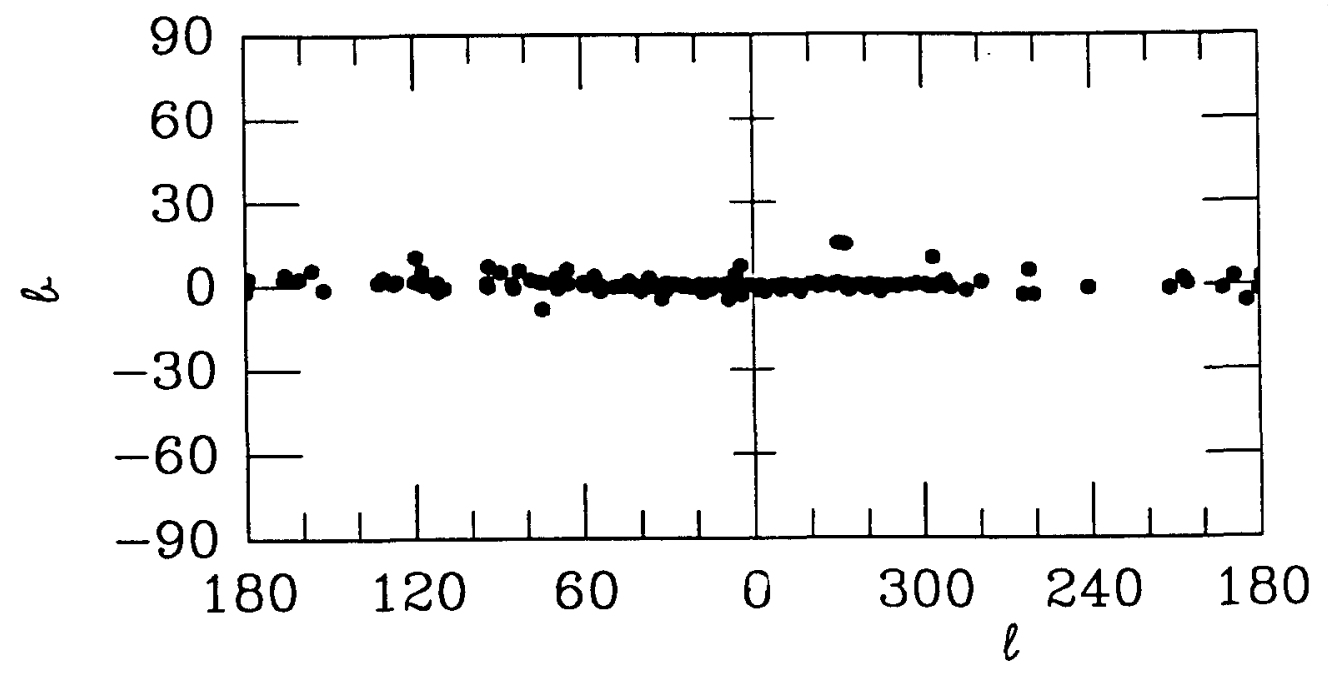

FIgURE 1. The distribution of the 182 known Galactic SNRs shown in Galactic coordinates.

TABLE 1. Statistics of Galactic supernova remnants

\begin{tabular}{lll}
\hline \hline SNR or suspected SNR: & & 182 \\
Morphological type - & Shell: & $80 \%$ \\
& Filled: & $10 \%$ \\
& Combination: & $10 \%$ \\
\hline
\end{tabular}

\section{SNR phenomenology}

As noted above, both gas heating and cosmic ray acceleration occur in the blast wave which accompanies all known SNRs. The intimate association between these two processes is illustrated by detailed comparison of the (thermal) X-ray emission with the (nonthermal) radio structure in such well-studied remnants as Kepler (e.g. Matsui et al, 1984), Tycho (Seward, 1990; Dickel et al, 1991) and the Cygnus Loop (Seward, 1990; Green, 1990). In general, morphological details correlate to a striking extent, although the brightness distributions do not necessarily do so.

We can discern two basic kinds of SNR morphology from the radio maps: the rimbrightened shells, and the more amorphous centrally-peaked Crab-Nebula-like remnants; a linear superposition of the two provides a third variant. The existing catalogue shows that, to the extent that they can be morphologically classified (and deviations of various kinds, as well as the quality of existing maps, leave the nature of some SNRs in doubt), the majority of remnants fall into the shell category, while the remainder are split roughly equally between the Crab-like and a combination of the other two. Table 1 summarizes the classification status of the 182 known SNRs (Green, op. cit.). It must be emphasized that such classifications are based upon radio structure alone. Optical emission appears to be much less complete, because of the capriciousness of both the relevant emission mechanisms and interstellar extinction. At X-ray wavelengths (where selective absorption can also play a role), the energy band - and hence gas temperature - may be relevant. 
There are a number of radio shells which have centrally-brightened X-ray emission (e.g., W44, 3C 400.2, MSH 11-61A; see Seward, 1990)。Of course the majority of Galactic SNRs, having only been detected in the radio, must necessarily be classified on the basis of radio maps.

It is generally accepted that the Crab-type remnants owe their striking characteristics (morphology, synchrotron emission, flat spectrum), just like their renowned namesake, to power released as an energetic neutron star spins down. It is clear from recent discoveries of low-luminosity mini-Crab remnants associated with the pulsars PSR 1951+32 (SNR CTB 80; Strom, 1987), PSR 1757-24 (Milne 56; Frail \& Kulkarni, 1991) and others, that such objects cover a wide range of luminosities, although the central pulsar has yet to be seen in a number (like 3 C 58 and CTB 87). In any event, the center-filled remnants, constituting a minority of known SNRs, - though an important minority, it must be said, - and being the topic of one of the other discourses (by Chevalier), will not figure prominently in the rest of this review.

Before resuming the thread of my discussion of SNRs in general, however, a word of caution about the statistics reflected in Table 1 is in order. On the face of it, it would seem that only a minority of SNRs comprise neutron-star-driven nebulae, while the majority of supernovae are believed to leave behind neutron stars (see, e.g., Van den Bergh \& Tammann, 1991; and Van den Bergh's contribution to these proceedings). While it may be, as has often been conjectured, that the pulsar-nebulosity phase is relatively shortlived compared to shell SNRs, it is not improbable that the former are under-represented in existing catalogues. A thorough understanding of the numerous selection effects to which SNRs have been subjected is required before attempting statistical analyses of data such as contained in Table 1 .

One simple inference which can be drawn, although it too should be regarded with a degree of circumspection, has to do with the (radio) SNR content of the Galaxy. If the average age attained by SNRs before dissipation is as much as $100000 \mathrm{yr}$, and if supernovae occur at the rate of 2 per century (see Van den Bergh \& Tammann, 1991; and Van den Bergh, these proceedings), then the Milky Way should contain some 2000 remnants, about 10 times the number catalogued. If this age is an overestimate, and $20000 \mathrm{yr}$ is nearer to the mark, then we have discovered nearly half of the Galactic population. The truth probably lies somewhere between these two very approximate estimates.

Besides arming us to slice through the interstellar fog of dust and ionized gas (as well as providing an additional strategem for pinpointing shock fronts), what does the radio emission from SNRs tell us? In the first place, the very existence of (synchrotron) radiation indicates the presence of high energy cosmic rays and magnetic fields to an enhanced degree. The strength of this emission provides information about the (minimum) energy densities present, while its power law spectral index, derived from the energy spectrum of the emitting particles, relates to the acceleration process. Radiation losses will produce a spectral break whose frequency is related to the source age and magnetic field strength. The polarization enables us to probe the magnetic fields within the SNR emission, and radio techniques have provided estimates of the distances to a number of remnants. Let us begin with this last point, as we require distances to calculate many physical parameters.

\section{Determining remnant distances}

As usual in astronomy, distance determinations are fraught with a variety of uncertainties, and SNRs are no exception. There have been two widely used techniques based 
upon radio measurements, one of which can be said to be fairly reliable (though not without its difficulties and limitations), while the other should be regarded as having considerable shortcomings. In addition, there are several other purely radio techniques which can either be applied to only a few objects, or require a number of assumptions.

The most reliable distance determinations by a radio technique are based upon the kinematical distances to clouds which absorb radiation from the object in question. The so-called $\mathrm{HI}$ absorption technique (other radio lines - $\mathrm{OH}, \mathrm{H}_{2} \mathrm{CO}$ - can also be used, but are generally weaker) provides a lower limit to the remnant's distance, and is obviously only as good as our knowledge of Galactic kinematics. Because of irregular motions and line broadening due to internal dispersion, the technique gives greater fractional accuracy at larger distances; it is essentially useless for objects closer than $1 \mathrm{kpc}$. In directions where the Galaxy displays large kinematic anomalies, - the SNR Tycho provides an example (Albinson et al, 1986), - interpretation of the data is not straightforward. Moreover in the two inner quadrants, where the line of sight crosses the solar circle, the velocities are double-valued, while the technique breaks down toward the Galactic center and anticenter, where circular orbital motion is transverse to the line of sight.

The most dubious, though widely-used, technique is based on the presumed relationship between surface brightness and remnant diameter (or $\Sigma-D$ ). In its original form, it makes the tacit assumption that all remnants (or at least those of uniform morphological type) will have about the same radio luminosity at a given linear diameter. Despite refinements which attempt to account for distance from the Galactic plane (Caswell \& Lerche, 1979), the practical utility of the technique is limited at best (Berkhuijsen, 1986; Green, 1984). Moreover, as Green (1991) has shown, the "relationship", such as it is, arises to a considerable extent from a lack of low surface brightness remnants to which existing SNR surveys are insensitive anyway. What does seem to be a genuine effect, at least to the extent that it is not caused by such instrumental selection, is an upper envelope to the distribution of $\Sigma$ vs. $D$ : There appears to be a maximum $\Sigma$ which a SNR of given $D$ can attain. In this limited sense, the $\Sigma-D$ relationship can be used to set an upper limit to a remnant's distance, but it should never be forgotten that the actual distance could be as much as an order of magnitude smaller.

Another radio method for determining SNR distance is applicable to the minority of remnants associated with a pulsar. A pulsar's dispersion measure is related to its distance, which can then be derived. Such distances depend upon models of the electron density in the Milky Way (Taylor \& Cordes, 1993), and have an uncertainty of about 50 \%. Most of the remaining methods rely upon parameters derived from optical or X-ray observations.

\section{Physical parameters}

With distances for a significant number of SNRs, we are in a position to calculate their radio-derived properties. The simplest of these is the average diameter, which is based upon the angular diameters found in Green's (1991) latest compilation. Distances are also drawn from Green, although a few have been augmented from the general literature. A histogram showing how Galactic SNRs are distributed according to diameter is shown in Fig. 2 (upper), while a similar plot for remnants in the Magellanic Clouds appears in Fig. 2 (lower). The deficiency of small-diameter SNRs in the latter is probably due to the limited resolution with which candidate radio sources have been studied in the Clouds. Apart from this difference, the two distributions are roughly similar, suggesting that the Galactic distances used are fairly accurate. From the distances and $1 \mathrm{GHz}$ flux densities, it is also possible to calculate the monochromatic luminosities, and these are shown for the 

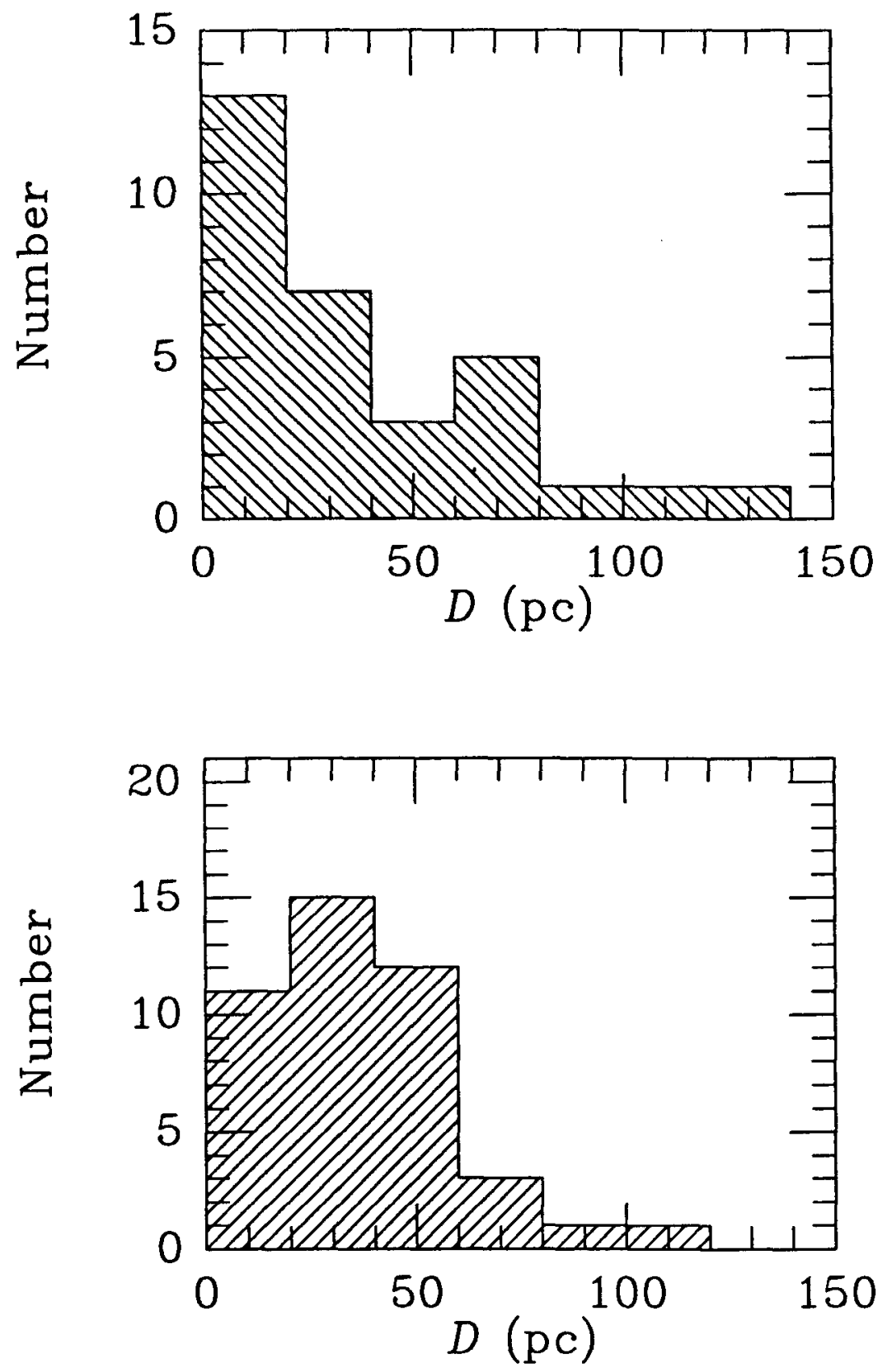

FIgURE 2. (upper) The diameters of Galactic SNRs with well-determined distances. (lower) The diameters of SNRs in the Large and Small Magellanic Clouds. The ordinates have been scaled to give the two histograms equal areas.

Galactic remnants in Fig. 3, where the median value is $L_{1 \mathrm{GHz}} \simeq 4 \times 10^{16} \mathrm{~W} \mathrm{~Hz}^{-1}$. (The distribution for SNRs in the Magellanic Clouds is similar, except that there are relatively fewer low luminosity remnants, as might be expected from a flux-limited sample.)

The rate at which synchrotron radiation is emitted from a source depends upon both 


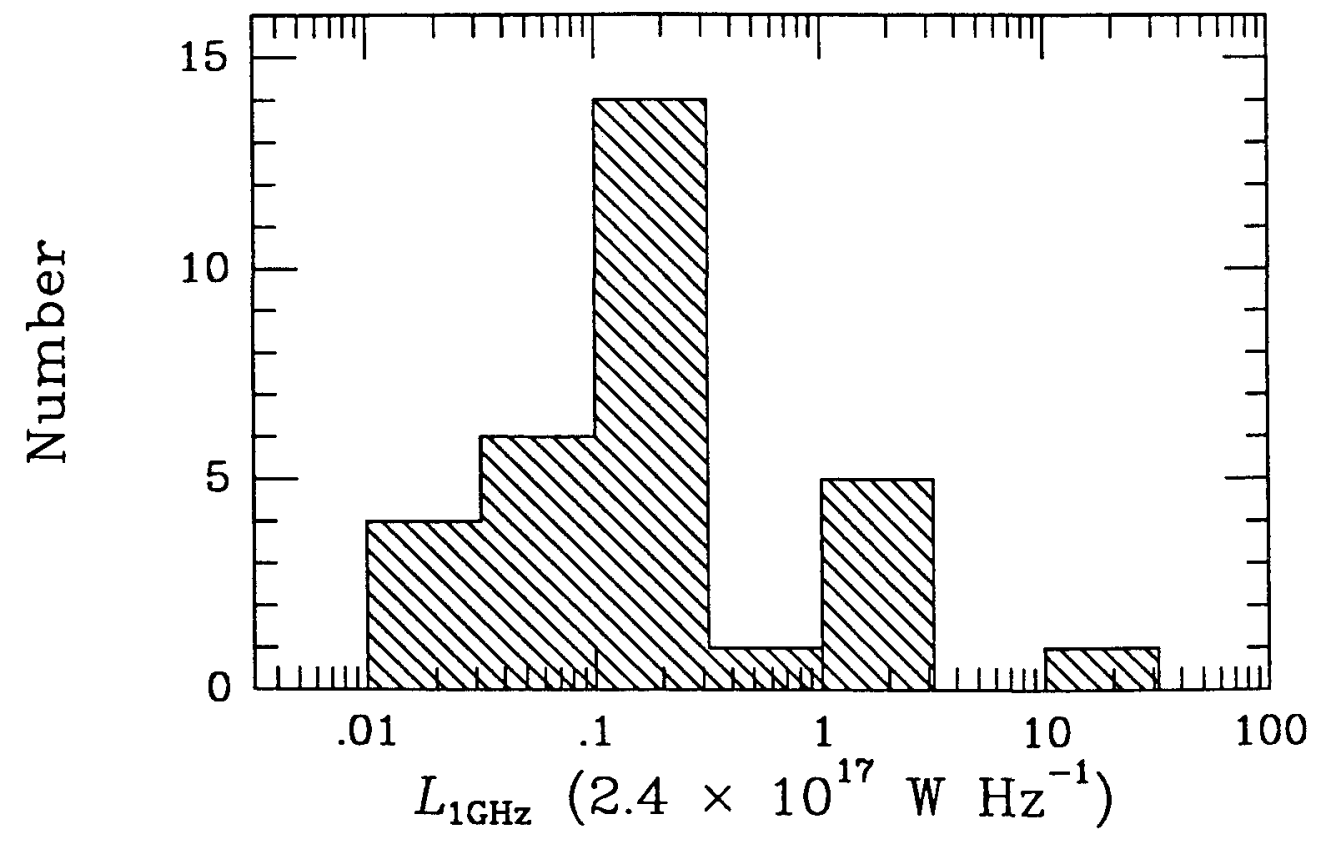

FIGURE 3. The $1 \mathrm{GHz}$ luminosities of Galactic SNRs with well-determined distances.

the particle energy, $E$, and the magnetic field strength, $B$ :

$$
\frac{\mathrm{d} E}{\mathrm{~d} t} \propto E^{2} B^{2}
$$

and of course the total power radiated is proportional to the number of emitting particles at a given energy, $N(E)$. Consequently, we need additional information about a source's magnetic field if we are to determine the amount of energy in cosmic rays. Clues about a remnant's magnetic properties are contained in the polarized component of its nonthermal emission.

Synchrotron radiation can be polarized by as much as $70 \%$, although field irregularities and projection effects usually reduce measured values considerably. The plane of the linearly polarized component at a sufficiently short wavelength can be used to deduce the magnetic field direction projected onto the sky. Low resolution maps of the magnetic field have been constructed in this way for over two-dozen remnants (see Milne, 1987), and they show that the shells tend to have either radially- or tangentially-directed fields. In all young (less than a few thousand years old) shell SNRs, the field is found to run radially. In larger, more mature remnants, the tendency is tangential, although there are objects where it is almost unidirectional, while in others it is complex. The degree of polarization at short wavelengths, which in most remnants is considerably less than the theoretical $\simeq 70 \%$, provides information about how much disorder there is.

Although the linear polarization does not directly depend upon the magnetic field strength, it can provide us with some information via the Faraday effect. The amount of Faraday rotation produced in a source depends upon the rotation measure, $\propto \int n_{\mathrm{e}} \mathbf{B} \cdot \mathrm{dl}$. If the electron density, $n_{\mathbf{e}}$, can be independently determined (for example, from X-ray observations), and the source depth (l) also, then some estimate of the magnetic field strength can be made. This technique has been applied to the young remnant of Kepler's 
supernova (Matsui et al, 1984), and the resulting field strength was found to be near equipartition.

Another way of determining the magnetic field strength is from the spectral break caused by radiation losses. If the initial maximum energy of particles is arbitrarily high, a simple integration of (4.1) shows that after a time $t_{1} \propto E_{1}^{-1} B^{-2}$, all of the original electrons will have energies $E<E_{1}$. Even if there is particle reacceleration, the spectrum will steepen above a frequency, $\nu_{\mathrm{b}}=1.33 \times 10^{16} B_{-5}^{-3} t_{4}^{-2} \mathrm{~Hz}$, where $B_{-5}$ is in units of $10 \mu \mathrm{G}$ and $t_{4}$ in $10^{4} \mathrm{yr}$. For $\nu_{\mathrm{b}}$ to fall within the radio regime, old objects with strong magnetic fields are required. The spectral break has been observed in the Crab Nebula, but at infrared wavelengths, where a time-averaged magnetic field strength of $440 \mu \mathrm{G}$ is inferred (Strom \& Greidanus, 1992). Infrared measurements in other SNRs like Cas A, where strong magnetic fields might be expected, are hampered by intense emission from shock-heated dust (e.g., Braun, 1987). A possible effect may have been detected in the flat spectrum remnant $3 \mathrm{C} 58$, although interpretation of the data is not straightforward (Green \& Scheuer, 1992). So determination of field strengths through the spectral break, which in any event requires an age estimate, is unlikely to be a realistic possibility in most SNRs.

For a final method of estimating magnetic field strengths, we return to polarization measurements, although now not of linear polarization but the much weaker circular variety. Incoherent synchrotron emission produces small amounts of circular polarization, the magnitude of which depends upon the magnetic field strength and frequency:

$$
\frac{V}{I} \simeq 0.1\left(\frac{B_{-4}}{\nu_{300}}\right)^{\frac{1}{2}} \%
$$

where $V$ and $I$ are the Stokes parameters for circular polarization and total intensity, and $\nu_{300}$ is the frequency in units of $300 \mathrm{MHz}$. Even at low frequencies, only sources with fairly substantial magnetic field strengths will produce measurable amounts of circular polarization. Recent $92 \mathrm{~cm}$ observations with the Westerbork Telescope suggest that Cas A may have magnetic fields of several hundred $\mu \mathrm{G}$, although the interpretation is by no means straightforward.

For several of the young, bright SNRs, the magnetic field strengths appear to lie within a small factor of the equipartition value. This is perhaps not too surprising, but since it seems to happen in some of the youngest remnants, there may be some hope that it holds true in the rest. The equipartition magnetic field strengths of several dozen SNRs range from just under $10^{-5} \mathrm{G}$, to somewhat over $10^{-4} \mathrm{G}$, with a median value of $3 \times 10^{-5}$ G. The minimum energies of the same remnants span $10^{48}-3 \times 10^{50}$ erg (median value of $3 \times 10^{49} \mathrm{erg}$ ). The smallest energies are associated with the most compact (youngest) remnants. Since they contain large kinetic energies, while the extended (but old and slowly expanding) SNRs have less kinetic energy, the distribution is consistent with a transfer of energy from gaseous motion to cosmic rays.

\section{Acknowledgements}

I am grateful to the IAU for providing me with a travel grant to attend Colloquium 145. The Westerbork Radio Observatory is operated by the Foundation for Research in Astronomy, which receives financial support from the Netherlands Organization for Scientific Research (NWO). 


\section{REFERENCES}

Albinson, J. S., Tuffs, R. J., Swinbank, E. \& Gull, S. F. (1986). MNRAS, 219, 427

Berkhuijsen, E. M. (1986). A\&A, 166, 257

Braun, R. (1987). A\&A, 171, 233

Caswell, J. L. \& Lerche, I. (1979). MNRAS, 187, 201

Dickel, J. R., Van Breugel, W. J. M. \& Strom, R. G. (1991). AJ, 101, 2151

Frail, D. A. \& Kulkarni, S. R. (1991). Nature, 352, 785

Green, D. A. (1984). MNRAS, 209, 449

Green, D. A. (1990). AJ, 100, 1927

Green, D. A. (1991). PASP, 103, 209

Green, D. A. \& Scheuer, P. A. G. (1992). MNRAS, 258, 833

Haslam, C. G. T., Salter, C. J., Stoffel, H. \& Wilson, W. E. (1982). A\&A, 47, 1

Matsui, Y., Long, K. S., Dickel, J. R. \& Greisen, E. W. (1984). ApJ, 287, 295

Milne, D. K. (1987). Aust. J. Phys., 40, 771

Reich, W., Fürst, E., Reich, P. \& Reif, K. (1990). AAS, 85, 633

Seward, F. D. (1990). ApJS, 73, 781

Strom, R. G. (1987). ApJ, 319, L103

Strom, R. G. \& Greidanus, H. (1992). Nature, 358, 654

Taylor, J. H. \& Cordes, J. M. (1993). ApJ, 411, 674

Van den Bergh, S. \& Tammann, G. A. (1991). ARA\&A, 29, 363 